KEK Preprint 98-195

\title{
Quantum Caustics in the Gaussian Slit Experiment
}

\author{
Kenichi Horie, Hitoshi Miyazaki and Izumi Tsutsui ${ }^{1}$ \\ Institute of Particle and Nuclear Studies \\ High Energy Accelerator Research Organization (KEK), Tanashi Branch \\ Tokyo 188-8501, Japan \\ Shogo TANimura ${ }^{2}$ \\ Department of Applied Mathematics and Physics \\ Graduate School of Informatics, Kyoto University \\ Kyoto 606-8501, Japan
}

\begin{abstract}
We study classical and quantum caustics for systems with quadratic Lagrangians of the form $L=\frac{1}{2} \dot{x}^{2}-\frac{1}{2} \lambda(t) x^{2}-\mu(t) x$. After deriving the transition amplitude on caustics in a closed form, we consider the Gaussian slit experiment and point out that the focusing around caustics is stabilized against initial momentum fluctuations by quantum effect.
\end{abstract}

PACS codes: 02.30.Hg; 03.65.-w; 03.65.Sq

Keywords: Caustics, Semiclassical Approximations

\footnotetext{
1 E-mail: tsutsui@tanashi.kek.jp

2 E-mail: tanimura@kuamp.kyoto-u.ac.jp
} 
1. Introduction. When a family of classical paths focuses, the envelope of the trajectories forms a focal region called caustics. The non-existence of paths or a coalescence of trajectories are interesting classical phenomena pertaining to caustics, which have been studied extensively in various fields of physics (see, e.g., [1]). Their consequences on quantum physics are, however, somewhat involved. This is mainly due to the fact that the powerful and commonly used semiclassical approximation scheme [2, 3] breaks down when caustics occur, since in this approach the transition amplitude is obtained by summing up fluctuations around (a finite number of) classical paths which are assumed to exist. So far there have appeared numerous works on the problem of quantum caustics. On one hand, a generalized prescription for the semiclassical approximation in the path-integral framework has been devised $[4,3]$, and on the other, a detailed quantum analysis for caustics in the presence of higher order terms (in the semiclassical expansion) — which smear out and so defuse the singularity at caustics — has been pursued $[4,5]$ (see also $[3,6,7,8]$ ).

In this letter, we wish to provide a simple method to derive a closed form of the transition kernel on caustics in the semiclassical approximation. We also mention some novel quantum aspects observed on and around caustics, namely, the concentration of amplitude on the focal point, observed in the Gaussian slit (gedanken-)experiment [9]. For simplicity we restrict ourselves to one dimensional systems without higher order terms focusing on systems governed by the quadratic Lagrangian of the form ${ }^{3}$

$$
L[x]=\frac{1}{2} \dot{x}^{2}-\frac{1}{2} \lambda(t) x^{2}-\mu(t) x,
$$

for which the semiclassical approximation is known to be exact. The transition amplitude between the two given endpoints $a, b \in \mathbb{R}$ during the interval $[0, T]$ reads $[10,11]$

$$
K(b, T ; a, 0)=\left(\frac{i}{2 \pi \hbar}\left|\frac{\partial^{2} I\left[x_{\mathrm{cl}}\right]}{\partial a \partial b}\right|\right)^{\frac{1}{2}} e^{\frac{i}{\hbar} I\left[x_{\mathrm{cl}}\right]-\frac{i \pi}{2} m(\lambda)}
$$

where $m(\lambda)$ is the Morse index of the harmonic potential $\lambda$ and $I\left[x_{\mathrm{cl}}\right]=\int_{0}^{T} d t L\left[x_{\mathrm{cl}}\right]$ is the action evaluated for the classical path $x_{\mathrm{cl}}(t)$ connecting the two endpoints $a, b$. Clearly, when the classical path ceases to exist, the prefactor $\left|\partial^{2} I\left[x_{\mathrm{cl}}\right] / \partial a \partial b\right|$ becomes singular and so does the transition kernel (1.2). We shall see that the kernel at the singularity, i.e., on caustics, can nevertheless be obtained in the modified prescription [3] by making use of the unitarity relation of the kernel, which improves the previous treatment [5] based

3 Note that any Lagrangian at most quadratic in position and velocity can be brought into this form by partial integration. We use the dot to denote time derivative $\dot{x}=d x / d t$, and put the mass $m$ of the particle unity. 
on an abstract setting. Further, our analysis of the Gaussian slit experiment reveals that the quantum effect suppresses the susceptibility of the focal concentration against initial momentum fluctuations.

2. Classical caustics. Let us first recapitulate the classical caustics (for a fuller account, see [12]). The equation of motion derived from (1.1) and satisfied by the classical solution $x_{\mathrm{cl}}$ takes the form,

$$
A_{\lambda} x_{\mathrm{cl}}(t)=\mu(t), \quad \text { where } \quad A_{\lambda}:=-\left[\frac{d^{2}}{d t^{2}}+\lambda(t)\right]
$$

If we let $x_{\mathrm{cl}}(p, t)$ be the solutions characterized by the initial momenta $\dot{x}(p, 0)=p$, then the Jacobi field $J(p, t):=\partial x_{\mathrm{cl}}(p, t) / \partial p$ is a solution of the homogeneous part of the equation of motion (2.1),

$$
A_{\lambda} J(p, t)=0
$$

satisfying $J(p, 0)=0$. Since the Jacobi field so defined gives a measure for the variation at later times under initial momentum fluctuations, we see that caustics (at $t=T$ ) occur when its final value vanishes $J(p, T)=0$. Note that, on account of the relation $\partial^{2} I\left[x_{\mathrm{cl}}\right] / \partial a \partial b=$ $-1 / J(p, T)$, the classical caustics implies quantum caustics as well, as seen in (1.2). Let $u, v$ be two linearly independent solutions of the homogeneous equation (2.2) subject to the initial conditions $u(0)=0, \dot{u}(0)=1$ and $v(0)=1, \dot{v}(0)=0$. (In terms of the Jacobi field, we have $u(t)=J(p, t) / \dot{J}(p, 0)$.) Then the general solution of the full equation (2.1) is given by

$$
x(t)=\alpha v(t)+\beta u(t)+s(t)
$$

where $s$ is a special solution of (2.1) satisfying $s(0)=0$. The constants $\alpha, \beta$ are determined from the initial position $x(0)$ and velocity $\dot{x}(0)$, respectively.

When considering the quantum transition amplitude in semiclassical expansion, it is important to know whether to a given Dirichlet boundary condition

$$
x(0)=a, \quad x(T)=b
$$

there exists a classical trajectory. Suppose first there is no caustic at $t=T$, that is, the Jacobi field $J(p, t)$ (and thus also $u(t)$ ) does not vanish at $t=T$. Then, by choosing $\alpha=a$ and $\beta=(b-a v(T)-s(T)) / u(T)$ in the general solution (2.3), one obtains the desired trajectory which is clearly unique. However, when caustics occur at $t=T$ (i.e., $J(p, T)=0)$, then the final position $x(T)$ turns out to be independent of the initial velocity 
$\dot{x}(0)$, and while $\alpha=a, \beta$ is left arbitrary since $u(T)=0$. Thus whatever the initial velocity may be, all paths starting from $x(0)=a$ ends at the final point

$$
x(T)=v(T) \cdot a+s(T) .
$$

If the given value $b$ does not match this unique value, then there is no classical trajectroy satisfying the Dirichlet boundary condition (2.4).

This phenomenon can be considered as a special case of caustics in geometric optics (see e.g., [3]), and when it occurs we shall call the potential $\lambda$ critical, otherwise noncritical. For those critical potentials, it is convenient to introduce the constant

$$
k(\lambda):=\frac{v(T)}{v(0)}
$$

where here $v$ can be any solution of the homogeneous part $(2.2)$ with $v(0) \neq 0$. It is easy to show that $k(\lambda)$ is indeed independent of $v$, and in view of (2.5), it gives the stretching factor during the period $[0, T]$. The final point $x(T)$ in $(2.5)$ is called the conjugate point to $a$ or focal point.

If $\lambda$ is critical, then besides the stretching factor $k(\lambda)$, a further characteristics is given by the Morse index, which we now briefly outline. Let $I[x]$ be the action functional for paths $x$ with fixed boundary condition (2.4). The second variation of the action $\delta^{2} I$ around a given classical path $x_{\mathrm{cl}}(t)$ (which in case of critical potential $\lambda$ is assumed to exist) is a symmetric bilinear functional of the perturbations around $x_{\mathrm{cl}}$, and its kernel is given by the operator $A_{\lambda}$ in (2.1). From the consideration of Sturm-Liouville problem the operator $A_{\lambda}$ is known to possess a complete set of orthonormal eigenfunctions $u_{n}(t)[13,14]$ :

$$
A_{\lambda} u_{n}(t)=-\left[\frac{d^{2}}{d t^{2}}+\lambda(t)\right] u_{n}(t)=E_{n} u_{n}(t)
$$

with

$$
u_{n}(0)=u_{n}(T)=0 ; \quad \int_{0}^{T} d t u_{n}(t) u_{m}(t)=\delta_{n m} .
$$

The index of the bilinear functional (Hessian) $\delta^{2} I$ is given by the number of negative eigenvalues of eigenvalue problem (2.7) for non-degenerate $A_{\lambda}$ for which no zero mode exists. It characterizes the type of the saddle point of the action functional $I[x]$ at the extremum path $x_{\mathrm{cl}}$. (In this letter, we assume the eigenvalues to be bounded from below, which is for example the case when $\lambda$ is bounded from above.) The index is known to be equal to the Morse index which is the number of zeros of the Jacobi field on the half-open interval $(0, T][15]$ and denoted by $m(\lambda)$. Note that the bilinear functional $A_{\lambda}$ becomes 
degenerate if there arises a zero mode solution of (2.7), a $u_{m}$ with $E_{m}=0$ for some $m$, i.e., when caustics occur. In this case the Morse index gives the number of negative eigenvalues plus one, and accordingly the index of $A_{\lambda}$ may be extended even to this degenerate case by saying that it is given by the number of modes with $E_{n} \leq 0$.

3. Quantum caustics. We now study the quantum dynamics of the system (1.1). Let $I[x]$ be the action evaluated along a path fulfilling the boundary condition (2.4). Corresponding to this boundary condition (2.4), the transition amplitude in the path-integral is given by

$$
K(b, T ; a, 0)=\int_{x(0)=a}^{x(T)=b} \mathcal{D} x e^{\frac{i}{\hbar} I[x]} .
$$

In carrying out the path-integration, we need to take into account the fact that, when caustics occur, there may not exist a classical solution that respects the given boundary condition. The general case, including the caustic one, may be handled by the following procedure $[3,4]$.

Let $\bar{x}_{\mathrm{cl}}$ be a classical solution of the equation of motion (2.1) with the initial value $\bar{x}_{\mathrm{cl}}(0)=a$, and let $c:=\bar{x}_{\mathrm{cl}}(T)$ be the endpoint of the solution. Note that in general $c$ may not be equal to $b$, but if there is a solution having $b$ as its endpoint, then we shall omit the bar and denote this special solution by $x_{\mathrm{cl}}$. Such a solution exists if $\lambda$ is non-critical, whereas if $\lambda$ is critical the endpoint is determined uniquely $c=k(\lambda) a+s(T)$, see $(2.5)$ and (2.6). Now, let us introduce a fixed, smooth function $\rho(t)$ satisfying $\rho(0)=0$ and $\rho(T)=b-c$. Then any path obeying the boundary condition (2.4) may be decomposed as

$$
x(t)=\bar{x}_{\mathrm{cl}}(t)+\rho(t)+\eta(t)
$$

with $\eta(t)$ being the function representing the fluctuations vanishing at both of the ends $\eta(0)=\eta(T)=0$. With the help of the orthonormal eigenfunctions in (2.7) and (2.8) it may be expanded as $\eta(t)=\sum_{n} a_{n} u_{n}(t)$. Using this and the decomposition (3.2) we find by partial integrations

$$
\begin{aligned}
I\left[\bar{x}_{\mathrm{cl}}+\rho+\eta\right]=I & {\left[\bar{x}_{\mathrm{cl}}\right]+\left.I[\rho]\right|_{\mu=0}+\dot{\bar{x}}_{\mathrm{cl}}(T) \rho(T) } \\
& +\frac{1}{2} \sum_{n} E_{n} a_{n}^{2}+\rho(T) \sum_{n} a_{n} \dot{u}_{n}(T)+\sum_{n} E_{n} a_{n} \int_{0}^{T} d t \rho(t) u_{n}(t) .
\end{aligned}
$$

In order to perform the path-integral (3.1) we change the integral variables $\mathcal{D} x=\mathcal{D} \eta=$ $\mathcal{N} \prod_{n} d a_{n}$ where $\mathcal{N}$ is a Jacobian factor. Further, we replace $E_{n}$ with $E_{n}+i \epsilon, \epsilon>0$ 
infinitesimal, such that the integrations over $a_{n}$ become all Gaussian even if an eigenvalue $E_{m}$ should vanish. With these preparations one obtains

$$
K(b, T ; a, 0)=\mathcal{N}\left[\prod_{n}\left(E_{n}+i \epsilon\right)\right]^{-\frac{1}{2}} e^{\frac{i}{\hbar} \Phi(b, a ; \lambda)}
$$

where

$$
\begin{aligned}
\Phi(b, a ; \lambda):=I\left[\bar{x}_{\mathrm{cl}}\right]+\left.I[\rho]\right|_{\mu=0}+\dot{\bar{x}}_{\mathrm{cl}}(T) \rho(T) \\
\quad-\frac{1}{2} \sum_{n} \frac{1}{\left(E_{n}+i \epsilon\right)}\left\{\rho(T) \dot{u}_{n}(T)+\left(E_{n}+i \epsilon\right) \int_{0}^{T} d t \rho(t) u_{n}(t)\right\}^{2} .
\end{aligned}
$$

The kernel formula (3.4) is valid for any potential $\lambda$. Now for $\lambda$ non-critical, each $E_{n} \neq 0$, and in the limit $\epsilon \rightarrow 0$ the kernel (3.4) reduces to

$$
K(b, T ; a, 0)=\mathcal{N}\left[\prod_{n} E_{n}\right]^{-\frac{1}{2}} e^{\frac{i}{\hbar} I\left[x_{\mathrm{cl}}\right]}
$$

where $\rho(t)$ has been set identically zero in (3.5) owing to the existence of the solution $x_{\mathrm{cl}}$. The obtained kernel $(3.6)$ can be shown $[10,11]$ to be equivalent to $(1.2)$.

If, on the other hand, $\lambda(t)$ is critical, then $E_{m}=0$ for some $m$, and the integration over $a_{m}$ yields in the limit $\epsilon \rightarrow 0$ the delta-function $\delta\left(\rho(T) \dot{u}_{m}(T)\right)$ on account of the identity, $\lim _{\epsilon \rightarrow 0} \frac{1}{\sqrt{2 \pi \epsilon}} e^{-x^{2} / 2 \epsilon}=\delta(x)$. Hence, for critical $\lambda(t)$ the kernel formula (3.4) reduces to

$$
K(b, T ; a, 0)=\sqrt{\frac{2 \pi}{i}} \mathcal{N}\left[\prod_{n \neq m} E_{n}\right]^{-\frac{1}{2}} \delta\left(\rho(T) \dot{u}_{m}(T)\right) e^{\frac{i}{\hbar} I\left[x_{\mathrm{cl}}\right]} .
$$

In (3.7) we have set $\rho(t)=0$ in the phase part of the kernel (and hence we put $I\left[\bar{x}_{\mathrm{cl}}\right]=$ $I\left[x_{\mathrm{cl}}\right]$ ), which is allowed due to the delta-function and the fact $\dot{u}_{m}(T) \neq 0$. (This follows since both $u_{m}(T)=0$ and $\dot{u}_{m}(T)=0$ would imply $u_{m}=0$ identically.) We note that

$$
\rho(T)=b-k(\lambda) a-s(T)
$$

and, therefore, the kernel (3.7) clearly shows that classically forbidden transitions to nonconjugate points, $b \neq k(\lambda) a+s(T)$ from $a$, remains forbidden even quantum mechanically.

The remarkable fact in the case of caustics is that one can express the transition kernel (3.7) in terms of the stretching factor $k(\lambda)$ and the Morse index $m(\lambda)$. To see this, let us write the kernel (3.7) in the polar form,

$$
K(b, T ; a, 0)=R(T) \delta(\rho(T)) e^{i \Theta(T)} .
$$


Noting that the transition kernel $K(b, T ; a, 0)$ can be expressed as $\langle b|\widehat{U}(T, 0)| a\rangle$ in terms of time evolution operator $\widehat{U}(T, 0)$, the unitarity relation,

$$
\int d b K^{*}(b, T ; c, 0) K(b, T ; a, 0)=\int d b\left\langle c\left|\widehat{U}^{\dagger}(T, 0)\right| b\right\rangle\langle b|\widehat{U}(T, 0)| a\rangle=\delta(a-c)
$$

determines the modulus $R(T)$ to be $R(T)=\sqrt{|k(\lambda)|}$. On the other hand, since the Morse index gives the number of negative modes plus one, we have $\left[\prod_{n \neq m} E_{n}\right]^{-\frac{1}{2}}=$ $\left[\prod_{n \neq m}\left|E_{n}\right|\right]^{-\frac{1}{2}} e^{-\frac{i \pi}{2}(m(\lambda)-1)}$. Thus the phase part is given by $\Theta(T)=\frac{1}{\hbar} I\left[x_{\mathrm{cl}}\right]-\frac{\pi}{2}(m(\lambda)-1)$. By combining these we find that the transition kernel (3.7) takes the closed form in terms of the stretching factor and Morse index,

$$
K(b, T ; a, 0)=\sqrt{|k(\lambda)|} \delta(b-k(\lambda) a-s(T)) e^{\frac{i}{\hbar} I\left[x_{\mathrm{cl}}\right]-\frac{i \pi}{2} m(\lambda)},
$$

up to an overall constant of unit modulus. This final form agrees with the expression obtained earlier based on a different construction of the path-integral [5].

As application, take, for example, the forced harmonic oscillator given by $\lambda(t)=\omega^{2}$ and $\mu(t)=-f(t)$. A special solution satisfying $s(0)=0$ is then

$$
s(t)=\frac{1}{\omega} \int_{0}^{t} d t^{\prime} \sin \omega\left(t-t^{\prime}\right) f\left(t^{\prime}\right)
$$

For the present case, caustics occur at $\omega=\frac{n \pi}{T}$ with $n=1,2, \ldots$, where we have $k(\lambda)=$ $(-1)^{n}$ and $m(\lambda)=n$, respectively. The classical trajectory starting from $x_{\mathrm{cl}}(0)=a$ and ending up at the point (2.5) possesses the action,

$$
I\left[x_{\mathrm{cl}}\right]=a \int_{0}^{T} d t \cos \omega t f(t)-\frac{1}{\omega} \int_{0}^{T} d t \int_{0}^{t} d t^{\prime} \cos \omega t \sin \omega t^{\prime} f(t) f\left(t^{\prime}\right) .
$$

Hence, in particular, for constant $f$ the kernel (3.11) reduces to

$$
K(b, T ; a, 0)=\delta\left(b-(-1)^{n} a-\left\{1-(-1)^{n}\right\} f / \omega^{2}\right) e^{\frac{i}{\hbar} f^{2} T / 2 \omega^{2}-i n \pi / 2} .
$$

This formula coincides with the previous results $[16,17,18]$ obtained by other indirect means $^{4}$ for the (forced) harmonic oscillator.

4. Gaussian slit experiment. For $\lambda$ non-critical but close to a critical $\bar{\lambda}$, one expects that there will be a concentration in the transition amplitude around the focal point

\footnotetext{
4 The result in [18] obtained for $f(t) \neq 0$ is marred by an error in the phase.
} 
$b=k(\bar{\lambda}) a+s(T)$ conjugate to a given initial point $a$. We analyze how the concentration takes place quantum mechanically, based on the Gaussian slit (gedanken) experiment [9].

Let there be an apparatus which emits a particle from the origin $x=0$ at time $t=-\tau$. To get a Gaussian distribution at $t=0$, we place a 'Gaussian slit' centered at $x=a$ with effective width (variance) $\sigma_{0}$, which is given by the Gaussian transmission factor

$$
G\left(x-a ; \sigma_{0}\right)=N \exp \left\{-\frac{(x-a)^{2}}{4 \sigma_{0}^{2}}\right\} .
$$

The wave function $\psi(x, 0)$ of the particle at $t=0$ is then furnished by the product of the free particle kernel $K_{0}(x, 0 ; 0,-\tau)=\sqrt{1 /(2 \pi i \hbar \tau)} e^{i x^{2} / 2 \hbar \tau}$ and the transmission factor. From the normalization condition $\int d x|\psi(x, 0)|^{2}=1$ we determine the constant $N$ and obtain

$$
\psi(x, 0)=G\left(x-a ; \sigma_{0}\right) K_{0}(x, 0 ; 0,-\tau)=\frac{1}{\left(2 \pi \sigma_{0}^{2}\right)^{\frac{1}{4}}} \exp \left\{-\frac{(x-a)^{2}}{4 \sigma_{0}^{2}}+\frac{i x^{2}}{2 \hbar \tau}\right\},
$$

which has the average momentum $p=\int d x \psi^{*}(x, 0)\left(-i \hbar \frac{d}{d x}\right) \psi(x, 0)=a / \tau$. Using the kernel (1.2), the wave function at $t=T$ is then given by

$$
\psi(y, T)=\int d x K(y, T ; x, 0) \psi(x, 0)
$$

To simplify the ongoing discussion let us assume first $\mu=0$ in (1.1). Then, the classical action $I\left[x_{\mathrm{cl}}\right](y, T ; x, 0)$ is a quadratic polynomial homogeneous in $x$ and $y$, because we have $I\left[x_{\mathrm{cl}}\right](c y, T ; c x, 0)=I\left[c x_{\mathrm{cl}}\right]=c^{2} I\left[x_{\mathrm{cl}}\right]=c^{2} I\left[x_{\mathrm{cl}}\right](y, T ; x, 0)$ for any constant $c$. Thus, with $A, B$ and $C$ some functions of $T$, we may write

$$
I\left[x_{\mathrm{cl}}\right](y, T ; x, 0)=A x^{2}+B x y+C y^{2} .
$$

Then it is straightforward to see that the wave function (4.3) at time $t=T$ has the form

$$
\psi(y, T)=\frac{1}{\left(2 \pi \sigma^{2}(T)\right)^{\frac{1}{4}}} \exp \left\{-\frac{\left(y-x_{\mathrm{cl}}(T)\right)^{2}}{4 \sigma^{2}(T)}+i(\text { phase })\right\},
$$

with

$$
x_{\mathrm{cl}}(T)=-\frac{1}{B}(2 a A+p)
$$

and

$$
\sigma(T)=\sigma_{0}\left\{\left(\frac{x_{\mathrm{cl}}(T)}{a}\right)^{2}+\left(\frac{\hbar}{2 \sigma_{0}^{2} B}\right)^{2}\right\}^{\frac{1}{2}}
$$


The second term in (4.7) represents the quantum effect whereas the first term is just the classical variance, since the variation in the initial position $\Delta x_{\mathrm{cl}}(0)=\sigma_{0}$ around the center $x_{\mathrm{cl}}(0)=a$ for the classical path results in the final variation $\Delta x_{\mathrm{cl}}(T)=\sigma_{0}\left|x_{\mathrm{cl}}(T)\right| / a=$ $\sigma_{0}|k(\lambda)|$. (Note that the initial momentum $p$ is linearly dependent on the initial position where the path goes through.) Thus, as one expects, the quantum effect always enhances the spread of the Gaussian distribution.

Viewed as a function of the initial variance $\sigma_{0}$, the final variance $\sigma(T)$ attains its minimum, i.e., the highest concentration of intensity

$$
\sigma_{\min }(T)=\left|\frac{\hbar x_{\mathrm{cl}}(T)}{a B}\right|^{\frac{1}{2}} \quad \text { at } \quad \sigma_{0}=\left|\frac{\hbar a}{2 B x_{\mathrm{cl}}(T)}\right|^{\frac{1}{2}}
$$

which is precisely the point where the quantum effect matches the classical variance. It then follows from (4.6) and (4.8) that, ideally, the infinite concentration $\sigma_{\min }(T)=0$ takes place at $B= \pm \infty$ or at $p=-2 a A$. The former case corresponds to the expected caustics, which occur when the variance (4.7) becomes purely classical $\sigma(T)=\sigma_{0}|k(\lambda)|$ for which the infinite concentration is obtained by letting $\sigma_{0} \rightarrow 0$. By contrast, in the latter case the variance becomes purely quantum mechanical, as all paths passing through the slit coalesce toward the origin $x=0$ at $t=T$. In fact, this case is again caustics occurring during the combined period $[-\tau, T]$ under the potential $\lambda(t)$ which vanishes for $t<0$, where the infinite concentration at the quantum level is achieved if we let $\sigma_{0} \rightarrow \infty$. It is obvious, however, that both of these concentrations in intensity are unstable, since a small fluctuation in the parameters $B$ or $p$ will generally bring the variance to a large value. Nevertheless, one could achieve a sharp peak in intensity by adjusting the initial variance along with the parameters according to (4.8).

An important quantity for characterizing the concentration is the susceptibility of the variance against initial momentum fluctuations. Exposing the momentum dependence of the variance explicit $\sigma(T)=\sigma(p, T)$, we use the following normalized quantity for the susceptibility,

$$
S(p, T):=\frac{a}{\sigma_{0}} \frac{\partial}{\partial p} \sigma(p, T) .
$$

The susceptibility of (4.7) reads

$$
S(p, T)=|J(p, T)|\left\{1+\left(\frac{\hbar a}{2 \sigma_{0}^{2} B x_{\mathrm{cl}}(T)}\right)^{2}\right\}^{-\frac{1}{2}},
$$

where $J(p, T)=-\left(\partial^{2} I\left[x_{\mathrm{cl}}\right] / \partial y \partial x\right)^{-1}=-1 / B$ is the Jacobi field for the classical action (4.4). In the classical limit $\hbar \rightarrow 0$, the susceptibility reduces to the (absolute value of the) 
Jacobi field. In the two cases mentioned above in which an infinite concentration can in principle be possible, the susceptibility vanishes $S(p, T)=0$ and hence the Gaussian wave packet becomes free from momentum fluctuations. What is interesting in (4.10) is that the quantum effect suppresses the susceptibility of the variation against initial momentum fluctuations.

The above features of the Gaussian wave packet persist even for $\mu \neq 0$, because then the classical action $I\left[x_{\mathrm{cl}}\right](y, T ; x, 0)$ acquires only linear and constant terms in addition to the quadratic terms in (4.4) as can be explicitly seen from the form of the general classical solution derived after eq. (2.4). Accordingly, the time evolution by the integral (4.3) is essentially unchanged. Semiclassically, this may also be the case for more general systems, not only for those with quadratic Lagrangians we considered, in view of the earlier study [19] which suggests that these features are a norm for a generic system in the limit $\hbar \rightarrow 0$.

As an example of the above discussion, consider again the harmonic oscillator $\lambda=\omega^{2}$ (but with $\mu=0$ ). ¿From the familiar classical action [9] one reads off that $A=C=$ $\omega / 2 \tan \omega T$ and $B=-\omega / \sin \omega T$. With the center of the distribution $x_{\mathrm{cl}}(T)=a \cos \omega T+$ $(p / \omega) \sin \omega T$, one finds the variance,

$$
\sigma(T)=\sigma_{0}\left\{\left(\cos \omega T+\frac{p}{a \omega} \sin \omega T\right)^{2}+\left(\frac{\hbar \sin \omega T}{2 \sigma_{0}^{2} \omega}\right)^{2}\right\}^{\frac{1}{2}}
$$

possessing its minimum $\sigma_{\min }(T)=\left|\hbar x_{\mathrm{cl}}(T) \sin \omega T / a \omega\right|^{\frac{1}{2}}$ at $\sigma_{0}=\left|\hbar a \sin \omega T / 2 \omega x_{\mathrm{cl}}(T)\right|^{\frac{1}{2}}$. One thus sees that an infinite concentration of the amplitude takes place either at $\omega=\frac{n \pi}{T}$ or $p=-a \omega / \tan \omega T$.

5. Conclusion. When caustics occur in one dimension, only those paths connecting the conjugate points are allowed classically, and the intrinsic features of caustics are characterized by the stretching factor $k(\lambda)$ and the Morse index $m(\lambda)$. In quantum mechanics the transition is still allowed only between conjugate points, and for systems with quadratic Lagrangians we derived the path-integral kernel for the amplitude in a closed form, expressed solely in terms of the stretching factor, the Morse index, and the action of (any of the) solution paths. As for the situation near caustics, the Gaussian slit experiment showed that, although the variance itself is enhanced at the quantum level, the susceptibility of the variance of the packet against initial momentum fluctuations is suppressed. This was derived by considering a quantum analogue of the Jacobi field, which reduces to the ordinary Jacobi field in the classical limit. Possible extensions of this work on quantum caustics, e.g., effects of higher order terms in the Lagrangian on the quantum susceptibility or related problems in higher dimensions, are currently under investigation [20]. 


\section{References}

[1] M.V. Berry, Adv. Phys. 25 (1976) 1.

[2] V.P. Maslov and M.V. Fedoriuk, "Semiclassical Approximation in Quantum Mechanics", D. Reidel Publ., London, 1981.

[3] L.S. Schulman, "Techniques and Applications of Path Integration", John Wiley \& Sons, New York, 1981.

[4] L.S. Schulman, in "Functional Integration and its Applications", A.M. Arthurs, ed., Clarendon Press, Oxford, 1975.

[5] C. DeWitt-Morette, Ann. Phys. 97 (1976) 367.

[6] C. DeWitt-Morette, A. Maheshwari and B. Nelson, Phys. Rep. 50C (1979) 256.

[7] C. DeWitt-Morette, B. Nelson and T.-R. Zhang, Phys. Rev. D28 (1983) 2526.

[8] G. Dangelmayr and W. Veit, Ann. Phys. 118 (1979) 108.

[9] R.P. Feynman and A.R. Hibbs, "Quantum Mechanics and Path Integrals", McGrawHill, New York, 1965.

[10] I.M. Gel'fand and A.M. Yaglom, J. Math. Phys. 1 (1960) 48.

[11] S. Levit and U. Smilansky, Proc. Amer. Math. Soc. 21 (1977) 299; Ann. Phys. 103 (1977) 198.

[12] K. Horie, H. Miyazaki, I. Tsutsui and S. Tanimura, Quantum Caustics for Systems with Quadratic Lagrangians, KEK Preprint 98-161, hep-th/9810156.

[13] R. Courant and D. Hilbert, "Methods of Mathematical Physics", Interscience Publishers, New York, 1953.

[14] P.M. Morse and H. Feshbach, "Methods of Theoretical Physics", McGraw-Hill Book Company, New York, 1953.

[15] J. Milnor, "Morse Theory", Princeton University Press, Princeton, 1963.

[16] J.-M. Souriau, in "Group Theoretical Methods in Physics", A. Janner, T. Janssen and M. Boon, eds., Lecture Notes in Physics, 50, Springer-Verlag, Berlin, 1976.

[17] P.A. Horváthy, Int. J. Theor. Phys. 13 (1979) 245.

[18] B.K. Cheng, Phys. Lett. 101A (1984) 464.

[19] G.A. Hagedorn, Commun. Math. Phys. 71 (1980) 77.

[20] K. Horie, H. Miyazaki and I. Tsutsui, Quantum Caustics for Systems with Quadratic Lagrangians in Multi-Dimensions, in preparation. 